\title{
A new approach in topology via elements of an ideal
}

\author{
ERDAL EKICI
}

\begin{abstract}
New approaches in topology or related branches of mathematics have contributed in a valuable way to the science, and have yielded various new topics for investigation. The main goal of this paper is to examine a new approach and so a new form of open sets via elements of an ideal. The concept of $\alpha_{I}^{\star}$-open sets is introduced and discussed.
\end{abstract}

\section{Introduction and preliminaries}

Elements of topology, generalized spaces, special spaces, etc have been considered as main subjects or main problems in several branches of mathematics. So, new approaches in topology or related branches of mathematics have contributed in a valuable way to the science, and have yielded various new topics for investigation. Such studies were performed, for example, in [1], [4]-[14], [18], [19]. The main goal of this paper is to examine a new approach and so a new form of open sets via elements of an ideal. The concept of $\alpha_{I}^{\star}$-open sets is introduced and examined.

In the present paper, topological spaces will be denoted by $\Im$ with the topology $\eta$. The closure and the interior of a subset $T$ of $\Im$ will be denoted by $\hat{\mathbf{c}}(T)$ and $\hat{\imath}(T)$, respectively.

Definition 1.1 (see [17]). A non-empty class $\mathcal{I}$ of subsets of a set $\Im$ is called an ideal on $\Im$ if the following properties hold:

(i) If $T \subseteq U \in \mathcal{I}$, then $T \in \mathcal{I}$.

(ii) If $T, U \in \mathcal{I}$, then $T \cup U \in \mathcal{I}$.

Definition 1.2 (see [17]). Suppose that $\mathcal{I}$ is an ideal on a topological space $(\Im, \eta)$. The following operator is called a local function of $T$

Received July 19, 2018.

2010 Mathematics Subject Classification. 54A05; 54A10.

Key words and phrases. $\alpha_{I}^{\star}$-open; $\alpha$-open; $\alpha_{I}^{\star}$-closed; ideal; $\alpha$ - $I$-open; $\star$-dense; $\star$-continuous.

http://dx.doi.org/10.12697/ACUTM.2019.23.09 
with respect to $\mathcal{I}$ and $\eta:(\cdot)^{\star}: P(\Im) \longrightarrow P(\Im), T^{\star}=\{\alpha \in \Im: T \cap U \notin$ $\mathcal{I}$ for every $U \in \eta$ with $\alpha \in U\}$, where $P(\Im)$ is the set of all subsets of $\Im$.

It is shown in [16] that $\hat{\mathbf{c}}^{\star}(T)=T \cup T^{\star}$ is a Kuratowski closure operator. The topology which is generated by $\hat{\mathbf{c}}^{\star}$ is denoted by $\eta^{\star}$ and called the

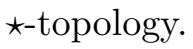

Definition 1.3. Let $\mathcal{I}$ be an ideal on a topological space $(\Im, \eta)$. A set $T$ in $(\Im, \eta)$ is said to be

(i) $\alpha$ - $I$-open $[15]$ if $T \subseteq \hat{\imath}\left(\hat{\mathbf{c}}^{\star}(\hat{\imath}(T))\right)$,

(ii) pre- $I$-open [2] if $T \subseteq \hat{\imath}\left(\hat{\mathbf{c}}^{\star}(T)\right)$,

(iii) $R$-I-open [20] if $T=\hat{\imath}\left(\hat{\mathbf{c}}^{\star}(T)\right)$,

(iv) $\star$-dense [3] if $\hat{\mathbf{c}}^{\star}(T)=\Im$.

\section{A new approach via elements of an ideal: $\alpha_{I}^{\star}$-open sets}

A new form of open sets, called $\alpha_{I}^{\star}$-open sets, is presented. The relationships and chracterizations of $\alpha_{I}^{\star}$-open sets are examined.

Definition 2.1. Let $\mathcal{I}$ be an ideal on a space $(\Im, \eta)$ and let $T$ be a set in $\Im$. A member $T$ of $\{U \subseteq \Im: U \neq \Im$ and there exists an open subset $V \neq \Im$ such that $\left.U \backslash \hat{\imath}\left(\hat{\mathbf{c}}^{\star}(V)\right) \in \mathcal{I}\right\} \cup\{\Im\}$ is called an $\alpha_{I}^{\star}$-open set.

Theorem 2.2. Let $\mathcal{I}$ be an ideal on a space $(\Im, \eta)$ and let $T$ be a set in $\Im$. Let $T$ be an $\alpha-I$-open set in $\Im$. Then $T$ is an $\alpha_{I}^{\star}$-open set in $\Im$.

Proof. Assume that $T$ is an $\alpha$ - $I$-open set. If we take $T=\Im$, then this implies that $T$ is an $\alpha_{I}^{\star}$-open set in $\Im$. Take $T \neq \Im$. This implies

$$
T \subseteq \hat{\imath}\left(\hat{\mathbf{c}}^{\star}(\hat{\imath}(T))\right) \text {. }
$$

If we put $S=\hat{\imath}(T)$, then we have $S \neq \Im$ and

$$
T \backslash \hat{\imath}\left(\hat{\mathbf{c}}^{\star}(S)\right) \in \mathcal{I} \text {. }
$$

Consequently, $T$ is an $\alpha_{I}^{\star}$-open set in $\Im$.

Corollary 2.3. Let $\mathcal{I}$ be an ideal on a space $(\Im, \eta)$ and let $T$ be a set in $\Im$. Suppose that $T$ is an open set in $\Im$. Then $T$ is an $\alpha_{I}^{\star}$-open set.

Proof. Every open set is $\alpha$-I-open. So, the claim follows from Theorem 2.2 .

Remark 2.4. Let $\mathcal{I}$ be an ideal on a space $(\Im, \eta)$ and let $T$ be a set in $\Im$. The following diagram holds for $T$ :

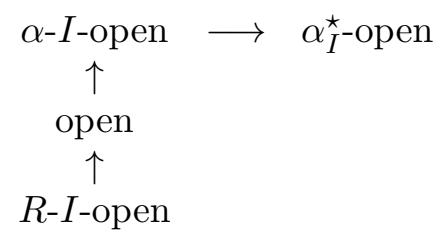


Remark 2.5. The implications in Remark 2.4 are not reversible in general. The example below shows such situation. The examples for the other implications can be found in the related papers.

Example 2.6. Let us take an ideal $\mathcal{I}=\{\emptyset,\{\theta\},\{\delta\},\{\theta, \delta\}\}$ on a topological space $\Im=\{\theta, \beta, \gamma, \delta\}, \eta=\{\Im,\{\theta\},\{\theta, \beta\},\{\gamma, \delta\},\{\theta, \gamma, \delta\}, \emptyset\}$. Take $T=\{\theta, \gamma\} \subseteq \Im$. Then $T=\{\theta, \gamma\} \subseteq \Im$ is an $\alpha_{I}^{\star}$-open set but $T$ is not $\alpha$-I-open.

Theorem 2.7. Let $\mathcal{I}$ be an ideal on a space $(\Im, \eta)$. A set $T$ in $\Im$ is $\alpha_{I^{\star}}^{\star}$ open if and only if either $T=\Im$ or there exist a set $S$ in $\mathcal{I}$ and an open set $W \neq \Im$ such that $T \backslash S \subseteq \hat{\imath}\left(\hat{\mathbf{c}}^{\star}(W)\right)$.

Necessity. Let $T$ be an $\alpha_{I}^{\star}$-open set in $\Im$. We have $T=\Im$ or $T \neq \Im$. Let $T \neq \Im$. This implies that there exists an open subset $W \neq \Im$ such that $T \backslash \hat{\imath}\left(\hat{\mathbf{c}}^{\star}(W)\right) \in \mathcal{I}$. If we take

$$
S=T \backslash \hat{\imath}\left(\hat{\mathbf{c}}^{\star}(W)\right),
$$

then we have $S \in \mathcal{I}$ and $T \backslash S \subseteq \hat{\imath}\left(\hat{\mathbf{c}}^{\star}(W)\right)$.

Sufficiency. Let $T=\Im$. Then $T$ is an $\alpha_{I}^{\star}$-open set in $\Im$. Suppose that there exist a set $S$ in $\mathcal{I}$ and an open subset $W \neq \Im$ such that $T \backslash S \subseteq \hat{\imath}\left(\hat{\mathbf{c}}^{\star}(W)\right)$. This implies

$$
T \backslash \hat{\imath}\left(\hat{\mathbf{c}}^{\star}(W)\right) \subseteq S .
$$

Since $T \backslash \hat{\imath}\left(\hat{\mathbf{c}}^{\star}(W)\right) \subseteq S$, one has $T \backslash \hat{\imath}\left(\hat{\mathbf{c}}^{\star}(W)\right) \in \mathcal{I}$. Thus, $T$ is an $\alpha_{I}^{\star}$-open set in $\Im$.

Theorem 2.8. Let $\mathcal{I}$ be an ideal on a space $(\Im, \eta)$ and let $T$ be a set in $\Im$. Then $T$ is $\alpha_{I}^{\star}$-open if and only if $T$ is a member of $\{U \subseteq \Im: U \neq \Im$ and there exist an open subset $S \neq \Im$ and $a V \in \mathcal{I}$ such that $\left.U \subseteq \hat{\imath}\left(\hat{\mathbf{c}}^{\star}(S)\right) \cup V\right\} \cup\{\Im\}$.

Proof. Let $T$ be an $\alpha_{I}^{\star}$-open set in $\Im$. We have $T=\Im$ or $T \neq \Im$. Let $T \neq \Im$. By Theorem 2.7, there exist an element $S$ of $\mathcal{I}$ and an open set $W \neq \Im$ such that $T \backslash S \subseteq \hat{\imath}\left(\hat{\mathbf{c}}^{\star}(W)\right)$. This implies $T \subseteq S \cup \hat{\imath}\left(\hat{\mathbf{c}}^{\star}(W)\right)$.

Theorem 2.9. Let $\mathcal{I}$ and $\hbar(\mathcal{I})=\{\hbar(\ell): \ell \in \mathcal{I}\}$ be ideals on topological spaces $(\Im, \eta)$ and $(\Re, \xi)$, respectively, where $\hbar: \Im \longrightarrow \Re$ is an open and $\star$-continuous bijection. For an $\alpha_{I}^{\star}$-open set $T$ in $\Im$, the set $\hbar(T)$ is $\alpha_{I}^{\star}$-open in $\Re$.

Proof. Suppose that $\hbar: \Im \longrightarrow \Re$ is an open $\star$-continuous bijection. Let $T$ be an $\alpha_{I}^{\star}$-open set in $\Im$. If $T=\Im$, then $\hbar(T)$ is an $\alpha_{I}^{\star}$-open set in $\Re$. Let $T \neq \Im$. This implies that there exists an open subset $S \neq \Im$ such that $T \backslash \hat{\imath}\left(\hat{\mathbf{c}}^{\star}(S)\right) \in \mathcal{I}$. We have $\hbar\left(T \backslash \hat{\imath}\left(\hat{\mathbf{c}}^{\star}(S)\right)\right) \in \hbar(\mathcal{I})$. This implies

$$
\hbar(T) \backslash \hbar\left(\hat{\imath}\left(\hat{\mathbf{c}}^{\star}(S)\right)\right) \in \hbar(\mathcal{I}) .
$$

Since $\hbar(T) \backslash \hat{\imath}\left(\hbar\left(\hat{\mathbf{c}}^{\star}(S)\right)\right) \subseteq \hbar(T) \backslash \hbar\left(\hat{\imath}\left(\hat{\mathbf{c}}^{\star}(S)\right)\right)$, we get $\hbar(T) \backslash \hat{\imath}\left(\hbar\left(\hat{\mathbf{c}}^{\star}(S)\right)\right) \in \hbar(\mathcal{I})$. Since

$$
\hbar(T) \backslash \hat{\imath}\left(\hat{\mathbf{c}}^{\star}(\hbar(S))\right) \subseteq \hbar(T) \backslash \hat{\imath}\left(\hbar\left(\hat{\mathbf{c}}^{\star}(S)\right)\right),
$$


we have

$$
\hbar(T) \backslash \hat{\imath}\left(\hat{\mathbf{c}}^{\star}(\hbar(S))\right) \in \hbar(\mathcal{I})
$$

So, $\hbar(T) \backslash \hat{\imath}\left(\hat{\mathbf{c}}^{\star}(\hbar(S))\right) \in \hbar(\mathcal{I})$. Consequently, $\hbar(T)$ is an $\alpha_{I}^{\star}$-open set in $\Re$.

Corollary 2.10. Let $\mathcal{I}$ and $\hbar(\mathcal{I})=\{\hbar(\ell): \ell \in \mathcal{I}\}$ be ideals on topological spaces $(\Im, \eta)$ and $(\Re, \xi)$, respectively, where $\hbar: \Im \longrightarrow \Re$ is a closed and $\star$-continuous bijection. For an $\alpha_{I}^{\star}$-open set $T$ in $\Im, \hbar(T)$ is an $\alpha_{I}^{\star}$-open set in $\Re$.

Proof. The claim follows from Theorem 2.9.

\section{Additional examinations}

The family of $\alpha_{I}^{\star}$-closed sets is presented. Chracterizations of $\alpha_{I}^{\star}$-closed sets and additional properties of $\alpha_{I}^{\star}$-open sets are examined.

Definition 3.1. Let $\mathcal{I}$ be an ideal on a space $(\Im, \eta)$ and let $T$ be a set in $\Im$. If $\Im \backslash T$ is an $\alpha_{I}^{\star}$-open subset, then $T$ is said to be $\alpha_{I}^{\star}$-closed.

Theorem 3.2. Let $\mathcal{I}$ be an ideal on a space $(\Im, \eta)$ and let $T$ be a set in $\Im$. Then $T$ is an $\alpha_{I}^{\star}$-closed set in $\Im$ if and only if either $T=\emptyset$ or there exist a set $S$ in $\mathcal{I}$ and a nonempty closed subset $V$ such that $\hat{\mathbf{c}}\left(\hat{\imath}^{\star}(V)\right) \backslash S \subseteq T$.

Necessity. Let $T$ be an $\alpha_{I}^{\star}$-closed subset of $\Im$. We have $T=\emptyset$ or $T \neq \emptyset$. Let $T \neq \emptyset$. Then we have $\Im \backslash T \neq \Im$ and also $\Im \backslash T$ is $\alpha_{I}^{\star}$-open. This implies that there exists an open subset $W \neq \Im$ such that

$$
(\Im \backslash T) \backslash \hat{\imath}\left(\hat{\mathbf{c}}^{\star}(W)\right) \in \mathcal{I} \text {. }
$$

If we take

$$
S=(\Im \backslash T) \backslash \hat{\imath}\left(\hat{\mathbf{c}}^{\star}(W)\right),
$$

then $S \in \mathcal{I}$ and $\Im \backslash T \subseteq \hat{\imath}\left(\hat{\mathbf{c}}^{\star}(W)\right) \cup S$. Since $\Im \backslash T \subseteq \hat{\imath}\left(\hat{\mathbf{c}}^{\star}(W)\right) \cup S$, one has

$$
\left(\Im \backslash \hat{\imath}\left(\hat{\mathbf{c}}^{\star}(W)\right)\right) \cap(\Im \backslash S) \subseteq T .
$$

If we take $V=\Im \backslash W$, it follows that $V$ is a closed set and $V \neq \emptyset$. This implies that the intersection of $\hat{\mathbf{c}}\left(\hat{\imath}^{\star}(V)\right)$ and $(\Im \backslash S)$ is a subset of $T$. Hence, $\hat{\mathbf{c}}\left(\hat{\imath}^{\star}(V)\right) \cap(\Im \backslash S) \subseteq T$.

Sufficiency. Let $T=\emptyset$. Then $T$ is an $\alpha_{I}^{\star}$-closed subset of $\Im$. Suppose that there exist a set $S$ in $\mathcal{I}$ and a nonempty closed set $V$ such that

$$
\hat{\mathbf{c}}\left(\hat{\imath}^{\star}(V)\right) \backslash S \subseteq T .
$$

We have $\Im \backslash T \subseteq\left(\Im \backslash \hat{\mathbf{c}}\left(\hat{\imath}^{\star}(V)\right) \cup S\right.$. If we take $W=\Im \backslash V$, then $W \neq \Im$ is an open subset. Since

$$
\Im \backslash T \subseteq\left(\Im \backslash \hat{\mathbf{c}}\left(\hat{\imath}^{\star}(V)\right) \cup S\right.
$$

we have

$$
\Im \backslash T \subseteq \hat{\imath}\left(\hat{\mathbf{c}}^{\star}(W)\right) \cup S
$$


Thus, $\Im \backslash T$ is an $\alpha_{I}^{\star}$-open subset of $\Im$ by Theorem 2.8. Consequently, $T$ is an $\alpha_{I}^{\star}$-closed subset of $\Im$.

Theorem 3.3. Let $\mathcal{I}$ be an ideal on a space $(\Im, \eta)$ and let $T$ be a set in $\Im$. Then $T$ is an $\alpha_{I}^{\star}$-closed set in $\Im$ if and only if either $T=\emptyset$ or there exists a closed subset $V \neq \emptyset$ such that $\hat{\mathbf{c}}\left(\hat{\imath}^{\star}(V)\right) \backslash T \in \mathcal{I}$.

Necessity. Let $T$ be an $\alpha_{I}^{\star}$-closed subset of $\Im$. By Theorem 3.2, there exist a set $S$ in $\mathcal{I}$ and a nonempty closed subset $V$ such that $\hat{\mathbf{c}}\left(\hat{\imath}^{\star}(V)\right) \cap(\Im \backslash S) \subseteq T$. This implies

$$
\hat{\mathbf{c}}\left(\hat{\imath}^{\star}(V)\right) \cap(\Im \backslash T) \subseteq S .
$$

Since $\hat{\mathbf{c}}\left(\hat{\imath}^{\star}(V)\right) \cap(\Im \backslash T) \subseteq S$, we have $\hat{\mathbf{c}}\left(\hat{\imath}^{\star}(V) \backslash T \in \mathcal{I}\right.$.

Sufficiency. If we take $T=\emptyset$, then $T$ is an $\alpha_{I}^{\star}$-closed subset of $\Im$. Suppose that there exists a nonempty closed set $V$ such that

$$
\hat{\mathbf{c}}\left(\hat{\imath}^{\star}(V)\right) \cap(\Im \backslash T) \in \mathcal{I} \text {. }
$$

Put $S=\hat{\mathbf{c}}\left(\hat{\imath}^{\star}(V)\right) \backslash T$. This implies that $S$ is an element of $\mathcal{I}$ and also, we

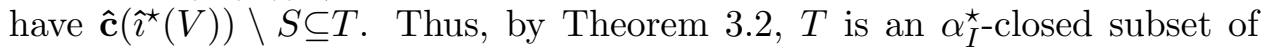
ऽ.

Theorem 3.4. Let $\mathcal{I}$ be an ideal on a space $(\Im, \eta)$ and let $T$ be a set in $\Im$. Then $T$ is an $\alpha_{I}^{\star}$-closed set in $\Im$ if and only if either $T=\emptyset$ or there exist a set $S$ in $\mathcal{I}$ and a closed subset $V \neq \emptyset$ such that $\hat{\mathbf{c}}\left(\hat{\imath}^{\star}(V)\right) \subseteq T \cup S$.

Necessity. Let $T$ be an $\alpha_{I}^{\star}$-closed subset of $\Im$. Suppose that $T \neq \emptyset$. Since $T$ is an $\alpha_{I}^{\star}$-closed subset of $\Im$, by Theorem 3.3, there exists a closed subset $V \neq \emptyset$ such that $\hat{\mathbf{c}}\left(\hat{\imath}^{\star}(V)\right) \backslash T \in \mathcal{I}$. If we take $S=\hat{\mathbf{c}}\left(\hat{\imath}^{\star}(V)\right) \backslash T$, then $S \in \mathcal{I}$, and we have

$$
\hat{\mathbf{c}}\left(\hat{\imath}^{\star}(V)\right) \subseteq T \cup S .
$$

Sufficiency. If we take $T=\emptyset$, then $T$ is an $\alpha_{I}^{\star}$-closed subset of $\Im$. Suppose that there exist $S \in \mathcal{I}$ and a closed set $V \neq \emptyset$ such that

$$
\hat{\mathbf{c}}\left(\hat{\imath}^{\star}(V)\right) \subseteq T \cup S .
$$

This implies $\hat{\mathbf{c}}\left(\hat{\imath}^{\star}(V)\right) \backslash T \subseteq S$. So, we have

$$
\hat{\mathbf{c}}\left(\hat{\imath}^{\star}(V)\right) \backslash T \in \mathcal{I} \text {. }
$$

By Theroem 3.3, $T$ is an $\alpha_{I}^{\star}$-closed set in $\Im$.

Theorem 3.5. Let $\mathcal{I}$ be an ideal on a space $(\Im, \eta)$. Suppose that there exists an open set $W$ such that $W \neq \Im$ and $W$ is $\star$-dense. Then every set in $\Im$ is an $\alpha_{I}^{\star}$-open subset.

Proof. Suppose that there exists an open set $W$ such that $W \neq \Im$ and $W$ is $\star$-dense.

Let $T$ be a subset of $\Im$. If we take $T=\Im$, then $T$ is an $\alpha_{I}^{\star}$-open subset. Let $T \neq \Im$. This implies $T \backslash \hat{\imath}\left(\hat{\mathbf{c}}^{\star}(W)\right) \in \mathcal{I}$. Hence, $T$ is an $\alpha_{I^{-}}^{\star}$-open subset. 
Theorem 3.6. Let $\mathcal{I}$ be an ideal on a space $(\Im, \eta)$ and let $T_{k}$ be an $\alpha_{I}^{\star}-$ open set in $\Im$ for $k \in \phi$. Then $\cap_{k \in \phi} T_{k}$ is an $\alpha_{I}^{\star}$-open subset of $\Im$.

Proof. Take $T_{k}=\Im$ for every $k \in \phi$. This implies that $\underset{k \in \phi}{\bigcap} T_{k}$ is an $\alpha_{I}^{\star}$-open subset of $\Im$.

Take $T_{k^{*}} \neq \Im$ for some $k^{*} \in \phi$. This implies that there exists an open set $S \neq \Im$ such that $T_{k^{*}} \backslash \hat{\imath}\left(\hat{\mathbf{c}}^{\star}(S)\right) \in \mathcal{I}$. We have $\underset{k \in \phi}{\cap} T_{k} \subseteq T_{k^{*}}$. It follows that $\left(\cap_{k \in \phi} T_{k}\right) \backslash \hat{\imath}\left(\hat{\mathbf{c}}^{\star}(S)\right) \subseteq T_{k^{*}} \backslash \hat{\imath}\left(\hat{\mathbf{c}}^{\star}(S)\right)$. Since $T_{k^{*}} \backslash \hat{\imath}\left(\hat{\mathbf{c}}^{\star}(S)\right) \in \mathcal{I}$, we get

$$
\left(\cap_{k \in \phi} T_{k}\right) \backslash \hat{\imath}\left(\hat{\mathbf{c}}^{\star}(S)\right) \in \mathcal{I} .
$$

Hence, $\underset{k \in \phi}{\cap} T_{k}$ is an $\alpha_{I}^{\star}$-open subset of $\Im$.

Remark 3.7. Let $\mathcal{I}$ be an ideal on a space $(\Im, \eta)$. The union of two $\alpha_{I}^{\star}$-open subsets need not be an $\alpha_{I}^{\star}$-open subset as shown in the example below.

Example 3.8. Take an ideal $\mathcal{I}=\{\emptyset,\{\theta\},\{\delta\},\{\theta, \delta\}\}$ on a topological space $\Im=\{\theta, \beta, \gamma, \delta\}, \eta=\{\Im,\{\theta\},\{\theta, \beta\},\{\gamma, \delta\},\{\theta, \gamma, \delta\}, \emptyset\}$. Take $T=$ $\{\theta, \gamma\}$ and $U=\{\beta\}$. Then $T$ and $U$ are $\alpha_{I}^{\star}$-open subsets but $T \cup U$ is not an $\alpha_{I}^{\star}$-open subset of $\Im$.

Theorem 3.9. Let $\mathcal{I}$ be an ideal on a space $(\Im, \eta)$. Assume that $T \neq \Im$ is a

Proof. Let $T \neq \Im$ be a $\star$-closed subset and $T \in \mathcal{I}$. This implies $T \backslash$ $\hat{\imath}\left(\hat{\mathbf{c}}^{\star}(T)\right)=T \backslash \hat{\imath}(T) \in \mathcal{I}$. If we take $S=\hat{\imath}\left(\hat{\mathbf{c}}^{\star}(T)\right)$, then $S$ is an open subset and $S \neq \Im$. Furthermore, we have

$$
T \backslash \hat{\imath}\left(\hat{\mathbf{c}}^{\star}(S)\right) \in \mathcal{I} .
$$

Thus, $T$ is an $\alpha_{I}^{\star}$-open subset.

Theorem 3.10. Let $\mathcal{I}$ be an ideal on a space $(\Im, \eta)$ and let $T \neq \Im$ be an $\alpha_{I}^{\star}$-open set. If $U \subseteq T$, then $U$ is $\alpha_{I}^{\star}$-open.

Proof. Assume that $T \neq \Im$ is an $\alpha_{I}^{\star}$-open subset and $U$ is a subset of $T$. This implies that there exists an open subset $S \neq \Im$ such that $T \backslash \hat{\imath}\left(\hat{\mathbf{c}}^{\star}(S)\right) \in$ $\mathcal{I}$. Since $U$ is a subset of $T$, we have

$$
U \backslash \hat{\imath}\left(\hat{\mathbf{c}}^{\star}(S)\right) \subseteq T \backslash \hat{\imath}\left(\hat{\mathbf{c}}^{\star}(S)\right) .
$$

Since $T \backslash \hat{\imath}\left(\hat{\mathbf{c}}^{\star}(S)\right) \in \mathcal{I}$, one has $U \backslash \hat{\imath}\left(\hat{\mathbf{c}}^{\star}(S)\right) \in \mathcal{I}$. Consequently, $U$ is an $\alpha_{I}^{\star}$-open set in $\Im$.

Theorem 3.11. Let $\mathcal{I}$ be an ideal on a space $(\Im, \eta)$ and let $T$ be a set in $\Im$. Assume that $T$ is a pre-I-open set and $T$ is not $\star$-dense in $\Im$. Then $T$ is $\alpha_{I}^{\star}$-open . 
Proof. Suppose that $T$ is a pre- $I$-open subset and $T$ is not $\star$-dense in $\Im$. This implies $T \subseteq \hat{\imath}\left(\hat{\mathbf{c}}^{\star}(T)\right)$ and $\hat{\mathbf{c}}^{\star}(T) \neq \Im$. We have

$$
\hat{\imath}\left(\hat{\mathbf{c}}^{\star}(T)\right) \neq \Im \text {. }
$$

If we take $V=\hat{\imath}\left(\hat{\mathbf{c}}^{\star}(T)\right)$, then we have $T \backslash \hat{\imath}\left(\hat{\mathbf{c}}^{\star}(V)\right) \in \mathcal{I}$. Consequently, $T$ is an $\alpha_{I}^{\star}$-open subset of $\Im$.

Remark 3.12. Let $\mathcal{I}$ be an ideal on a space $(\Im, \eta)$. The concepts of pre- $I$-openness and $\alpha_{I}^{\star}$-openness are independent as shown in the example below.

Example 3.13. Take an ideal $\mathcal{I}=\{\emptyset,\{\theta\},\{\delta\},\{\theta, \delta\}\}$ on a topological space $\Im=\{\theta, \beta, \gamma, \delta\}, \eta=\{\Im,\{\theta\},\{\theta, \beta\},\{\gamma, \delta\},\{\theta, \gamma, \delta\}, \emptyset\}$. Let $T=\{\beta\}$. Then $T$ is an $\alpha_{I}^{\star}$-open set but $T$ is not a pre- $I$-open set in $\Im$. On the other hand, $U=\{\theta, \beta, \gamma\}$ is a pre- $I$-open set but $U$ is not an $\alpha_{I}^{\star}$-open set.

\section{Acknowledgements}

The author would like to thank the referees. This work was supported by Çanakkale Onsekiz Mart University, The Scientific Research Coordination Unit, Project number: FYL-2017-1338.

\section{References}

[1] Á. Császár, Generalized open sets in generalized topologies, Acta Math. Hungar. 106 (2005), 53-66.

[2] J. Dontchev, Idealization of Ganster-Reilly decomposition theorems, arxiv:math. GN/9901017v1, 1999.

[3] J. Dontchev, M. Ganster, and D. Rose, Ideal resolvability, Topology Appl. 93 (1999), $1-16$.

[4] E. Ekici, Almost nearly continuous multifunctions, Acta Math. Univ. Comenian. 73(2) (2004), 175-186.

[5] E. Ekici, On $\gamma$-Urysohn spaces, Adv. Stud. Contemp. Math. 11(2) (2005), 219-226.

[6] E. Ekici, On $\mathcal{C}^{*}$-sets and decompositions of continuous and $\eta \zeta$-continuous functions, Acta Math. Hungar. 117(4) (2007), 325-333.

[7] E. Ekici, Generalization of weakly clopen and strongly $\theta$-b-continuous functions, Chaos Solitons Fractals 38 (2008), 79-88.

[8] E. Ekici, Generalized hyperconnectedness, Acta Math. Hungar. 133(1-2) (2011), 140147.

[9] E. Ekici, On $R$-I-open sets and $\mathcal{A}_{I}^{*}$-sets in ideal topological spaces, An. Univ. Craiova Ser. Mat. Inform. 38(2) (2011), 26-31.

[10] E. Ekici, On weak structures due to Császár, Acta Math. Hungar. 134(4) (2012), 565-570.

[11] E. Ekici, Further new generalized topologies via mixed constructions due to Császár, Math. Bohem. 140(1) (2015), 1-9.

[12] E. Ekici and T. Noiri, Decompositions of continuity, $\alpha$-continuity and $\mathcal{A B}$-continuity, Chaos Solitons Fractals 41 (2009), 2055-2061.

[13] E. Ekici and T. Noiri, «-extremally disconnected ideal topological spaces, Acta Math. Hungar. 122(1-2) (2009), 81-90. 
[14] E. Ekici and J. H. Park, On weakly s-precontinuous multifunctions, Arab. J. Sci. Eng. Sect. A Sci. 32(1A) (2007), 83-92.

[15] E. Hatir and T. Noiri, On decompositions of continuity via idealization, Acta Math. Hungar. 96 (2002), 341-349.

[16] D. Janković and T. R. Hamlett, New topologies from old via ideals, Amer. Math. Monthly 97 (1990), 295-310.

[17] K. Kuratowski, Topology, Vol. 1, Academic Press, New York, 1966.

[18] S. Modak and T. Noiri, Remarks on locally closed set, Acta Comment. Univ. Tartu. Math. 22(1) (2018), 57-64.

[19] T. Noiri and V. Popa, Minimal structures, punctually m-open functions in the sense of Kuratowski and bitopological spaces, Math. Commun. 12(2) (2007), 247-253.

[20] S. Yüksel, A. Açikgöz, and T. Noiri, On $\delta$-I-continuous functions, Turk J. Math. 29 (2005), 39-51.

Department of Mathematics, Çanakkale Onsekiz Mart University, TerzioĞLu CAmpus, 17020 ÇANAKKale/TURKEY

E-mail address: eekici@comu.edu.tr, prof.dr.erdalekici@gmail.com 